REDES-Revista hispana para el análisis de redes sociales

Vol.6,\#4, Jun./Jul. 2004

http://revista-redes. rediris.es

\title{
Redes variables en el tiempo: visualización con Pajek
}

\author{
Alejandro A. Ruiz León \\ Laboratorio de Redes-DMMSS \\ IIMAS-UNAM, MÉXICO \\ rarnulfo@servidor.unam.mx \\ Jorge Gil-Mendieta \\ Laboratorio de Redes-DMMSS \\ IIMAS-UNAM, MÉXICO \\ jgil@servidor.unam.mx
}

\section{Resumen}

Un tema de interés en el análisis de redes es el relacionado con la ocurrencia de cambios en una red. Nos interesa saber que pasa cuando se establecen nuevas relaciones entre los actores; cuando se rompen algunas de ellas; cuando se incluyen otros actores; cuando se excluye o cuando sale de la red un actor. También nos interesa conocer los cambios que ocurren en la red en el transcurso del tiempo y el significado de estos cambios.

En este ensayo mostramos la potencialidad del uso de Pajek en el análisis de redes variables en el tiempo, para ello nos referiremos a la parte del análisis funcional de la red de poder mexicana que hacen Schmidt y Gil-Mendieta (2002), donde analizan a 37 personajes de la vida política de México desde el año de 1920 hasta el año de 1990, haciendo cortes cada diez años.

\begin{abstract}
Our interest is to analyse a network trough time, how new relationships, new actors, the exclusion of actors, the exclusion of relationships impact the network from one period to another. This paper is based in the work of Schmidt and Gil (2002), where they analyse 37 Mexican political actors from 1920 to 1990, we use Pajek in order to visualize the network in periods of ten years.
\end{abstract}

Palabras clave: Redes variables en el tiempo, visualización 
REDES-Revista hispana para el análisis de redes sociales

Vol.6,\#4, Jun./Jul. 2004

http://revista-redes. rediris.es

\section{El núcleo de la red}

En su trabajo "La Red de Poder Mexicana", Schmidt y Gil-Mendieta abordan la problemática de entender las variaciones coyunturales del sistema, mediante el análisis funcional de la red a través del tiempo.

Los autores enfocan el estudio en el núcleo de la red, que en este caso consiste de 37 personajes de la vida política de México incluyendo a 17 ex-presidentes entre el año de 1920 y el año de 1990 (Anexo 1).

"... hay un núcleo de la red con una elevada concentración de recursos

y la posibilidad de movilizar su influencia ... "1

En la Figura 1 se muestran los 37 personajes que se consideraron para el estudio. En la parte central se localizan los ex-presidentes(nodos verdes) en forma cronológica empezando con Francisco I. Madero. Para el análisis longitudinal se tomaron periodos de diez años.

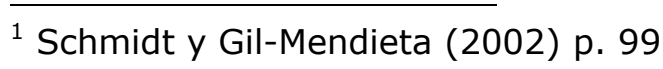




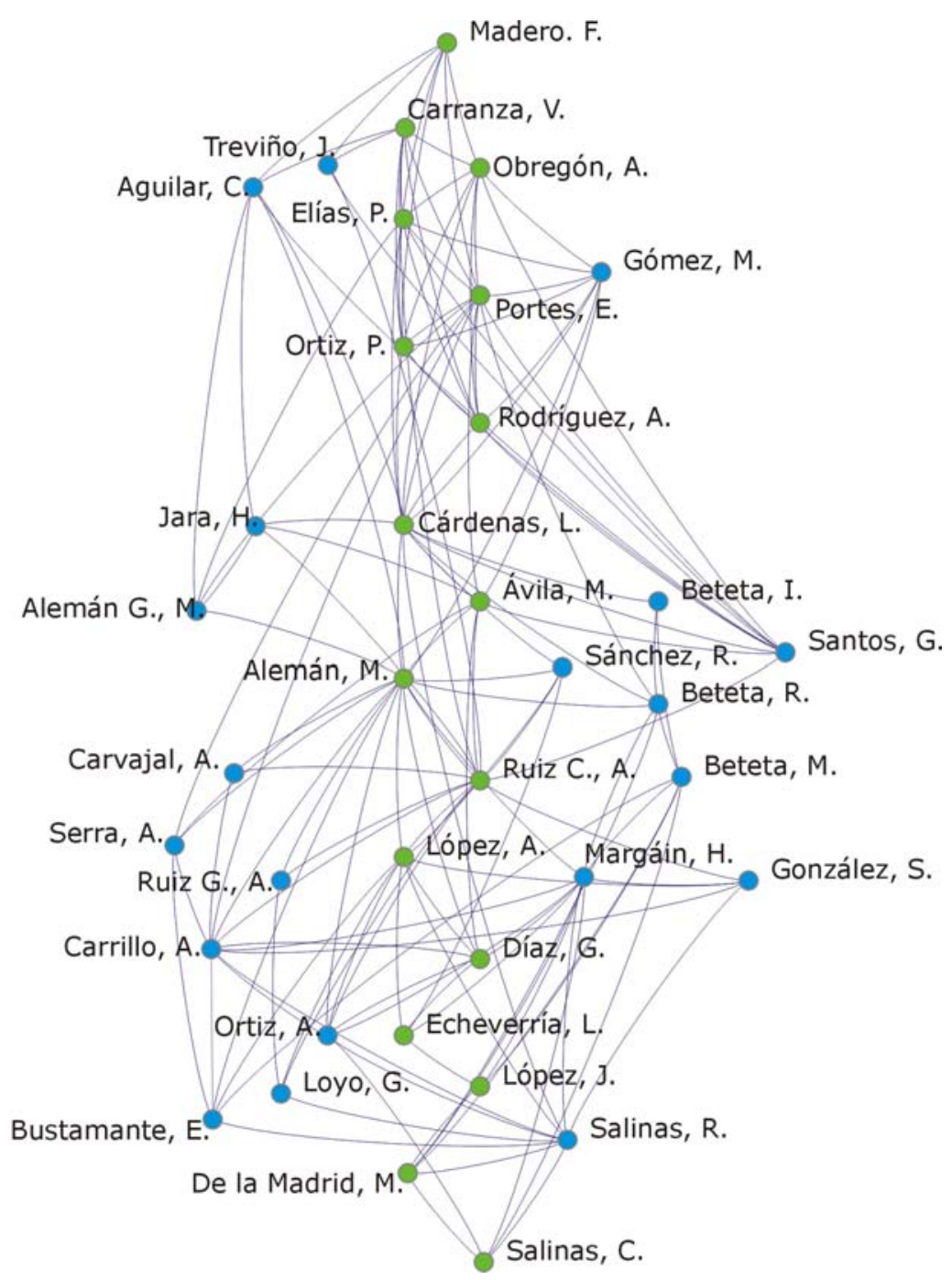

Figura 1.La red de poder mexicana

\section{Pajek}

Pajek maneja archivos .tim en los que se puede definir a un grado de detalle si un actor pertenece (esta activo) a la red en un determinado periodo y las propiedades (etiqueta, color, tamaño) con que se visualiza en ese periodo; lo mismo para las relaciones activas entre los actores.

Pajek también maneja una versión mas simplificada en que se define tanto para los actores como para las relaciones, los periodos en que están activos.

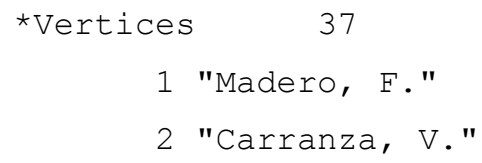




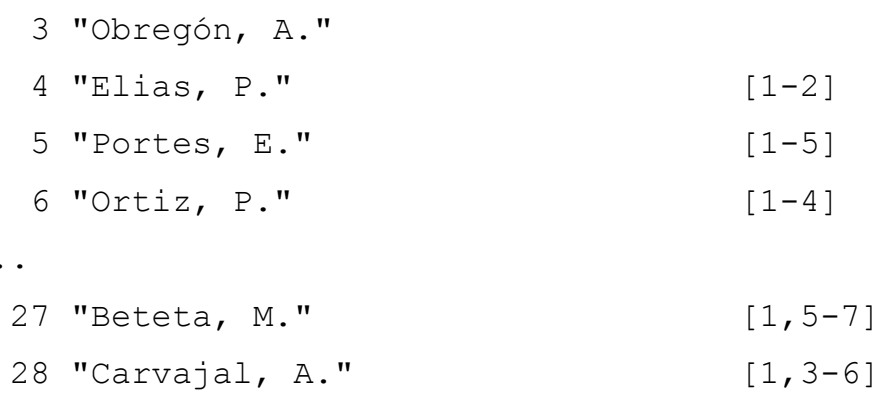

En el código anterior se esta indicando entre paréntesis cuadrados que el nodo 1 solo esta activo en el periodo 1, para el nodo 2 y 3 se aplica la misma indicación, para el nodo 5 se esta indicando que esta activo del periodo 1 al 5, para el nodo 27 se está indicando que esta activo el periodo 1 y del 5 al 7.

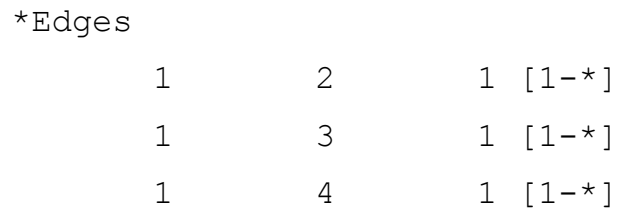

En el código anterior se esta indicando que las aristas están activas del periodo 1 al periodo final. En este caso se esta considerando que si el actor esta activo sus relaciones también, en caso de que las relaciones solo estén activas ciertos periodos habría que especificarlo de forma similar a las que se uso en el código para los nodos. Para cada una de las aristas hay que definir en que periodos están activas, lo mismo para cada arco.

El archivo lo creamos de la misma forma que un archivo .net y se carga a Pajek de la misma forma.

Para generar la red correspondiente a cada periodo se usó de la opción All en Net / Transform / Generate in Time / All. Figura 2 


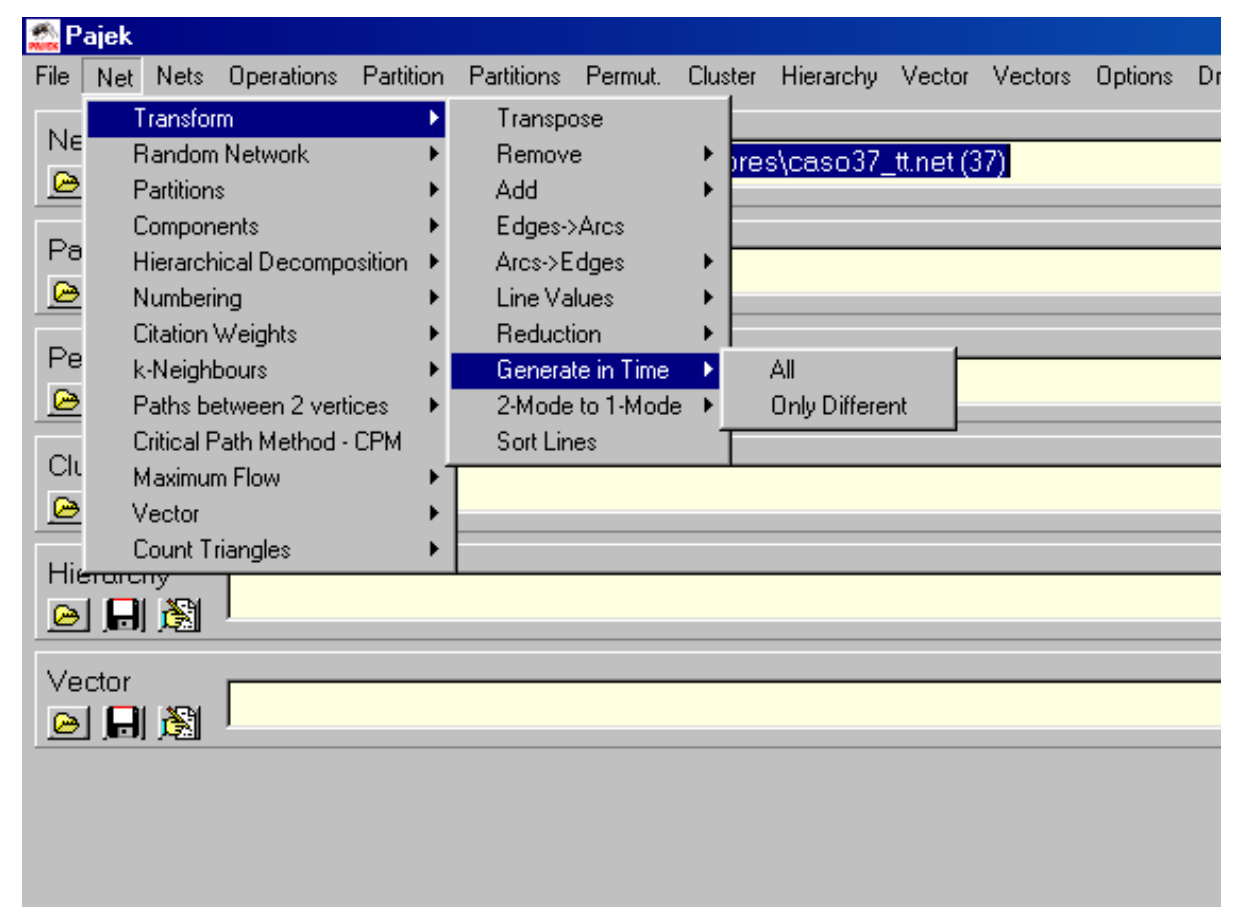

Figura 2.

Para visualizar las redes para cada periodo habría que seleccionarla como red activa y usar Draw, otra opción es visualizar la red activa usando Draw y cambiar de red activa usando Previous y Next. Figura 3.

Si para la red se definió un partición, Pajek genera una partición para cada una de la redes correspondientes a cada periodo. En este caso la partición se hizo tomando en cuenta a quienes fueron presidentes (nodos verdes) y quienes no fueron(nodo azules). Figura 3. 


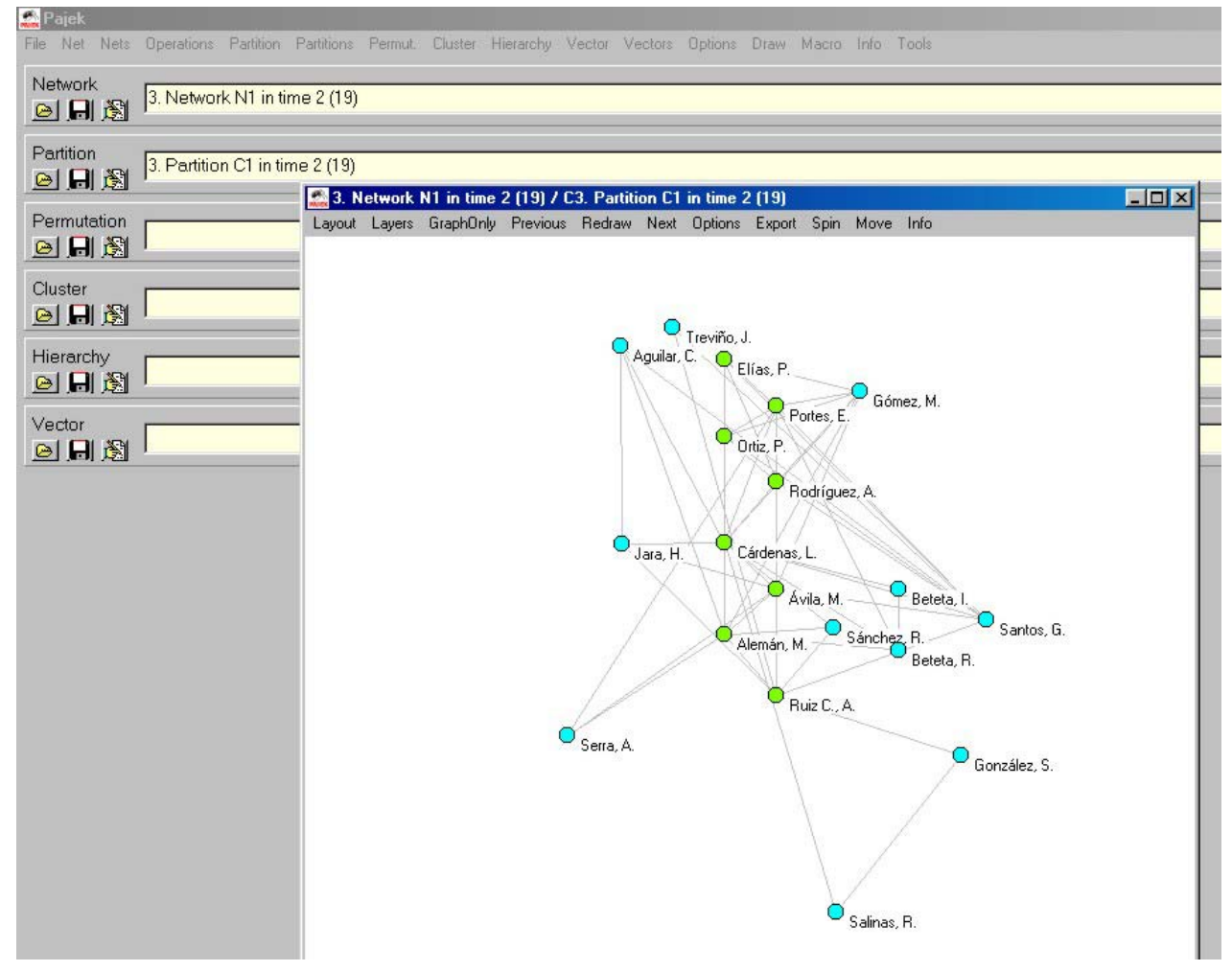

Figura 3. Ventana de visualización

\section{La red a lo largo del tiempo}

Los autores señalan que la red en que se incluyen todos los actores da información importante como la de "...las conexiones más importantes a lo largo del tiempo... "2, como son las relaciones de Lázaro Cárdenas Del Río, Miguel Alemán Valdés y Hugo B. Margáin Gleason. Estos tres personajes están relacionados con 33 elementos de la red, incluyéndolos, o sea cubren un $\mathbf{8 9 . 1 9 \%}$ y en el caso de incluir a Adolfo Ruiz Cortines cubrirían un $97.27 \%$ de la red. Hay que hacer notar que Adolfo Ruiz Cortines estuvo vinculado a Lázaro Cárdenas del Río y a Miguel Alemán Valdés, con el que no tuvo una vinculación directa fue con Hugo B. Margáin Gleason. Los cuatro personajes cubren 55 de los 143 vínculos establecidos.

En la Figura 4 se muestra la visualización de la matriz de co-pertenencia de los actores a los 60 cliques que se forman. Las aristas más gruesas indican una co-pertenencia a un número mayor de cliques.

${ }^{2}$ Ibíd. p. 106 


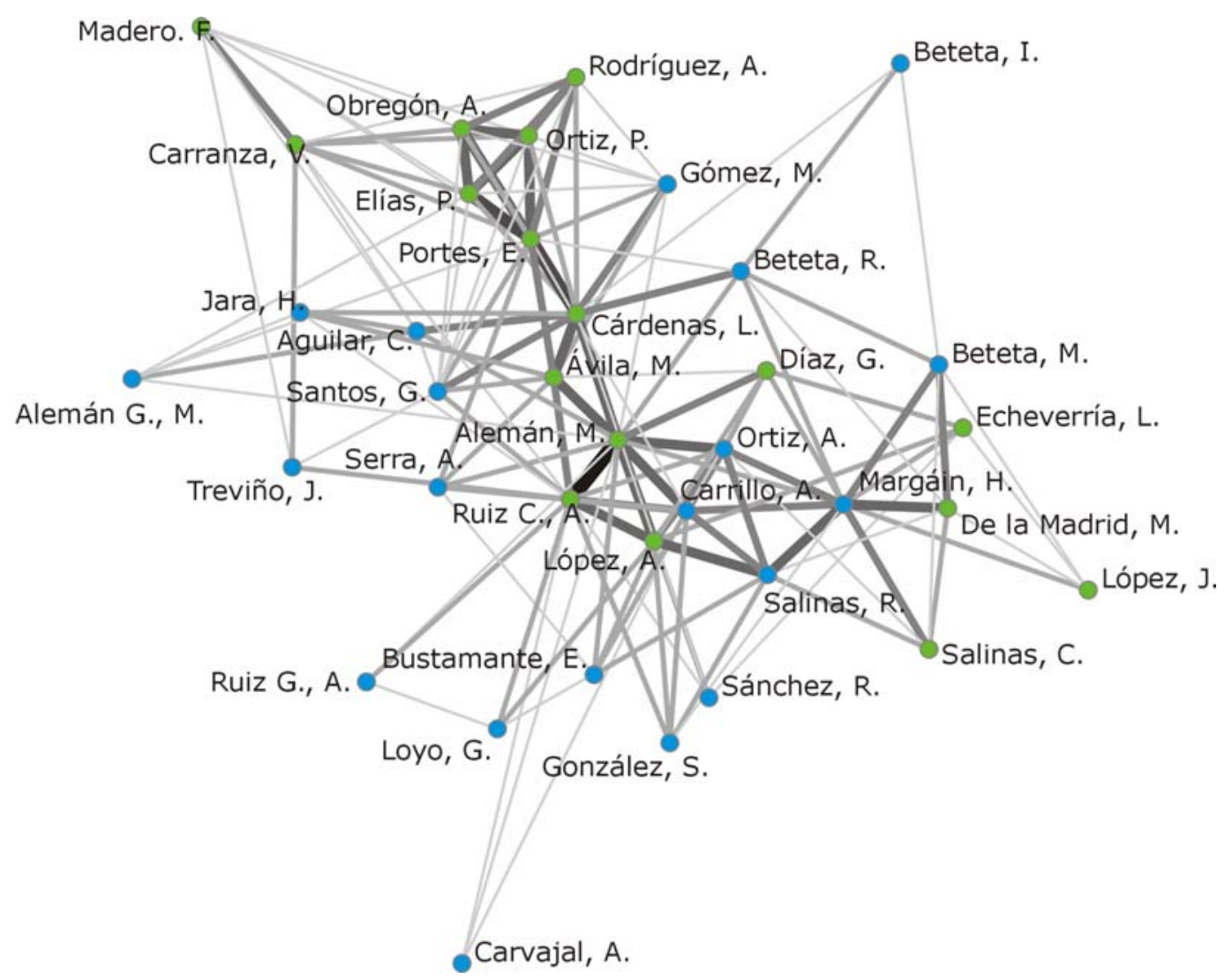

Figura 4. Visualización de la matriz de co-pertenencia a cliques

En la gráfica se puede observar un zona central caracterizada por aristas más gruesas, en esta zona encontramos a los actores con una mayor coparticipación en cliques, si fijamos como punto central a Miguel Alemán Valdés (Alemán, M.), observamos que en la parte izquierda de la gráfica la coparticipación en cliques entre los ex-presidentes es muy alta, mientras que en la parte derecha hay actores que no son ex-presidentes y tiene una coparticipación mayor a la que tienen los ex-presidentes, ejemplo Raúl Salinas Lozano (Salinas, R.) y Hubo B. Margáin Gleason (Margáin, H.).

\section{Los cambios a través del tiempo}

En las Figura $5 a, 5 b$ y $5 c$ es notoria la presencia de Miguel Alemán Valdés en la red, su influencia se muestra a partir del corte del año de 1940 hasta el corte del año de 1980, otros dos actores que destacan por su permanencia dentro de la red son Raúl Salinas Lozano y Salomón González Blanco. 


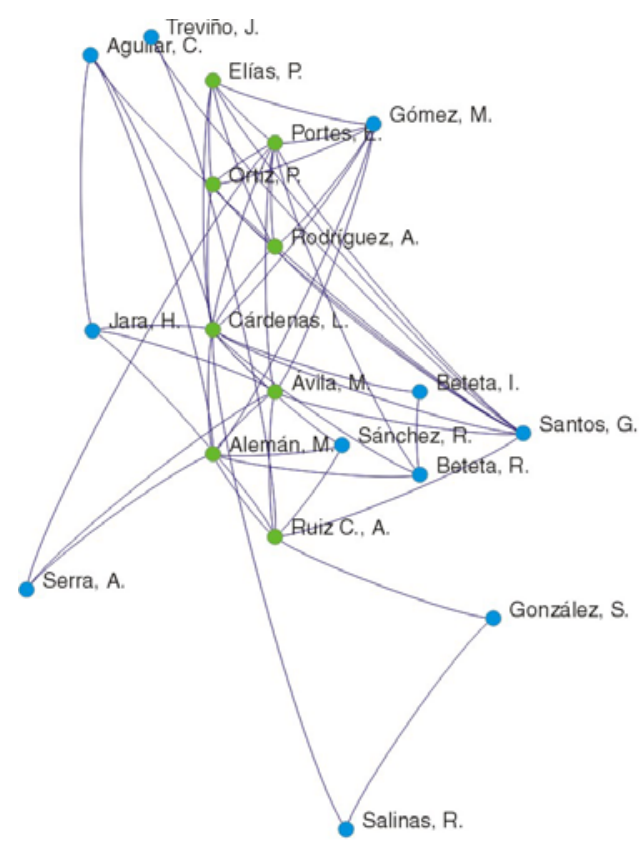

1940

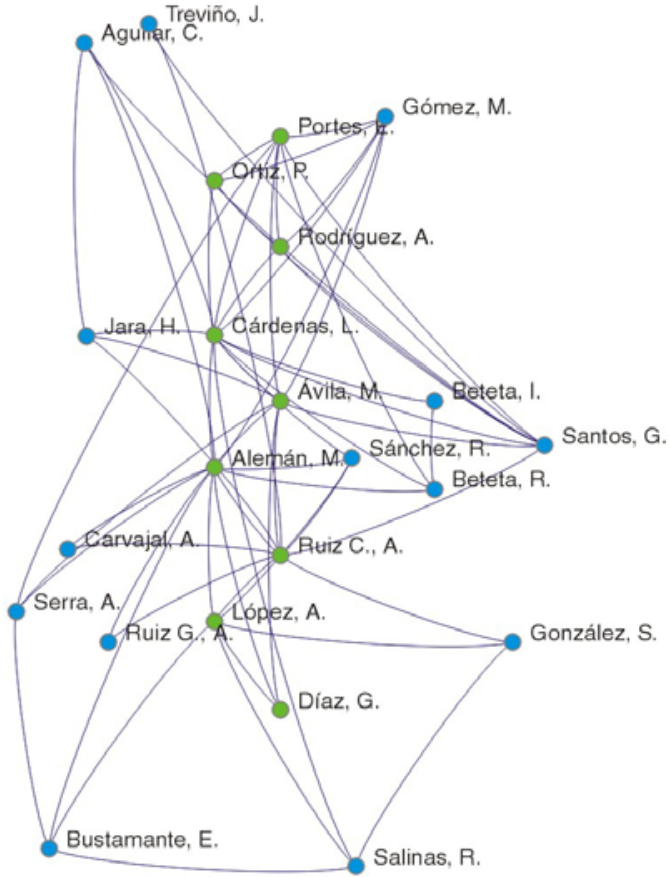

1950

Figura $5 a$. La red a través del tiempo

(ex presidentes-nodos de color verde)

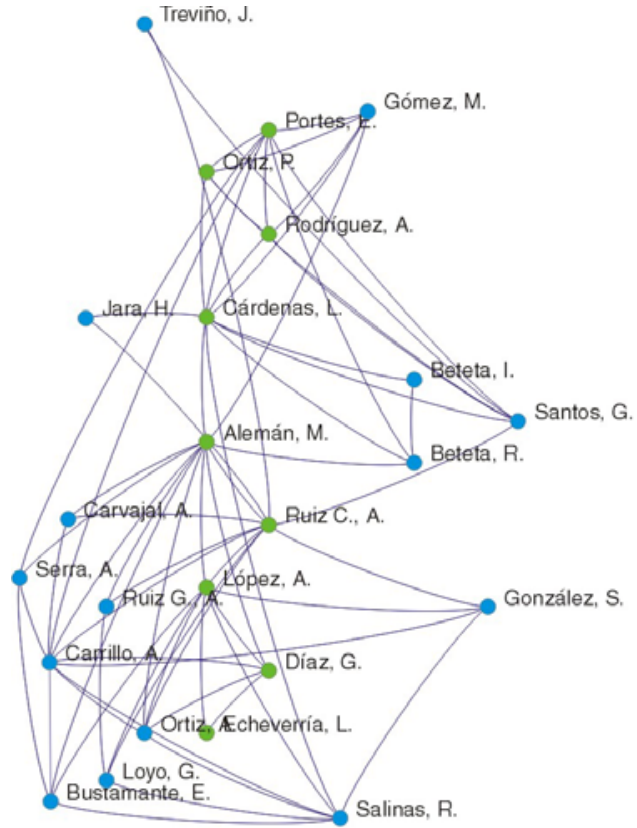

1960

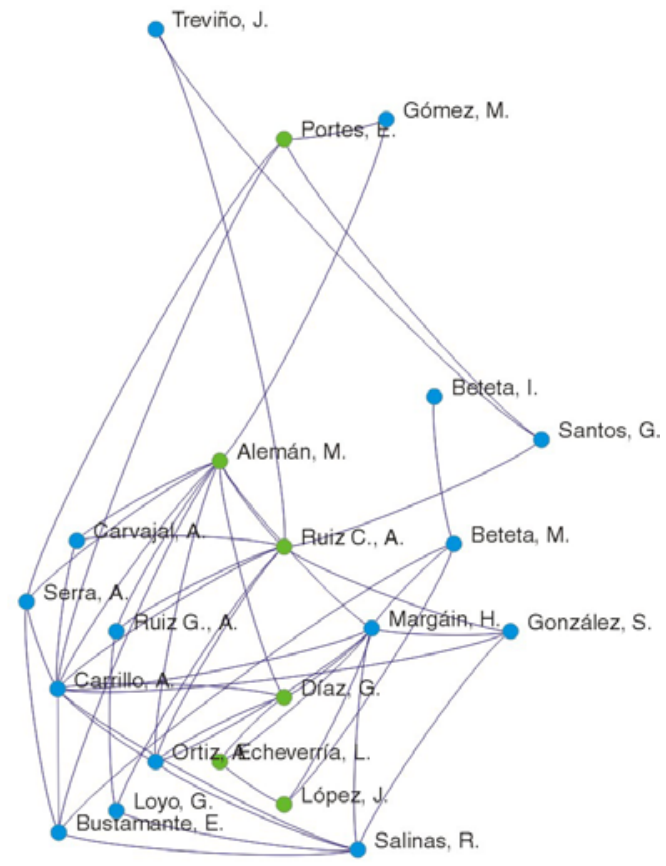

1970

Figura $5 \mathrm{~b}$. La red a través del tiempo (ex presidentes-nodos de color verde) 


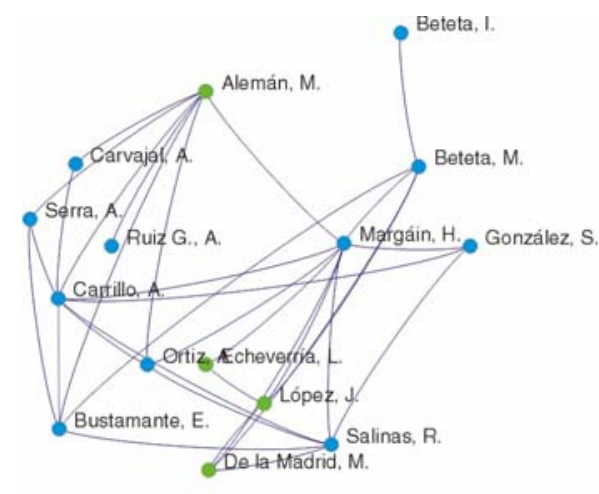

1980

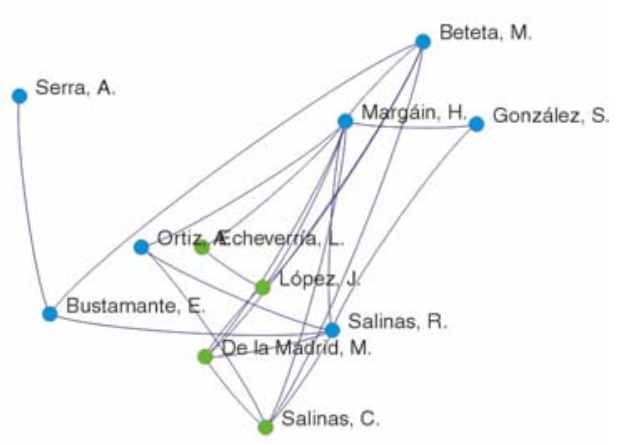

1990

Figura $5 c$. La red a través del tiempo (ex presidentes-nodos de color verde)

En su trabajo Schmidt y Gil-Mendieta señalan un cambio generacional que se acentúa con la salida de importantes líderes revolucionarios en la década de los años 1970's. En las figuras 6a, 6b y $6 c$ podemos ver que en el corte del año de 1970 están presentes solo tres actores del grupo de los militares (nodos rojos). Para la década de 1990 la red está formada solo por actores del grupo de civiles (nodos azules).

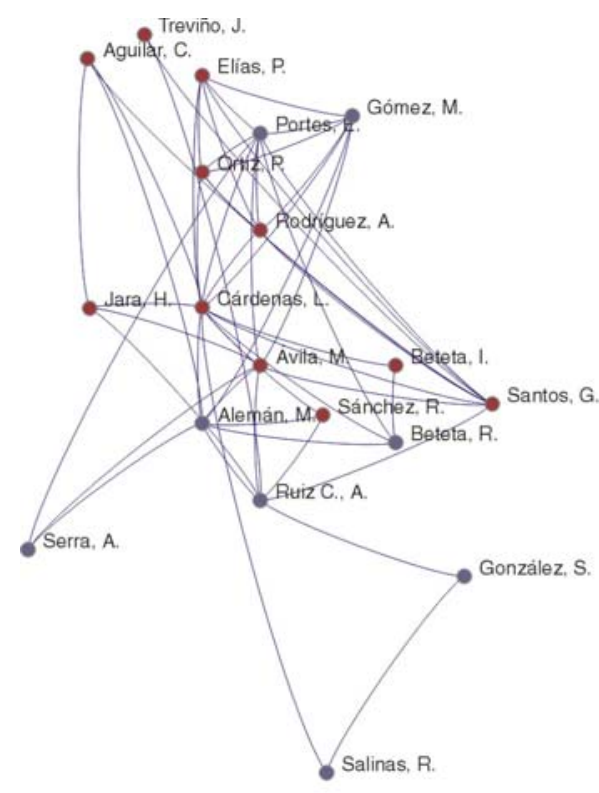

1940

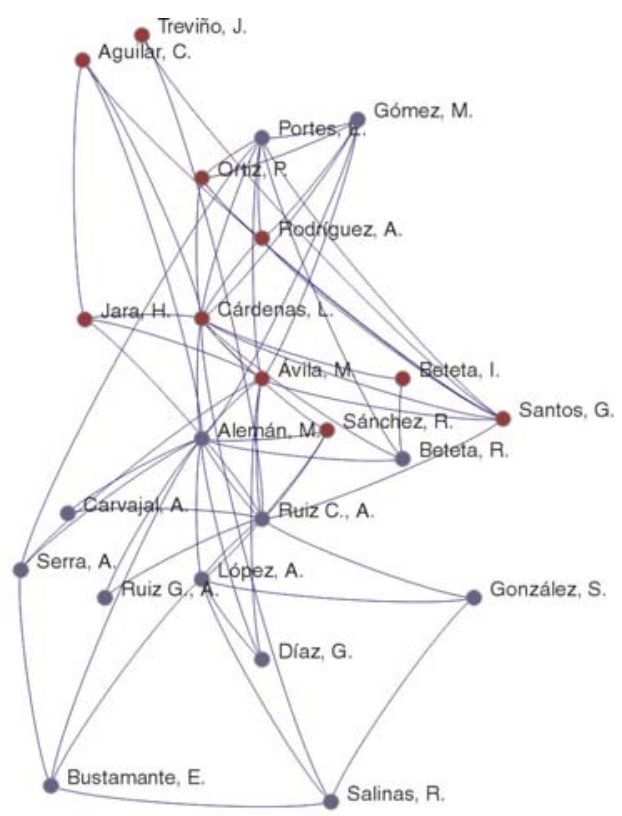

1950

Figura 6a. La red a través del tiempo (militares-nodos de color rojo) 


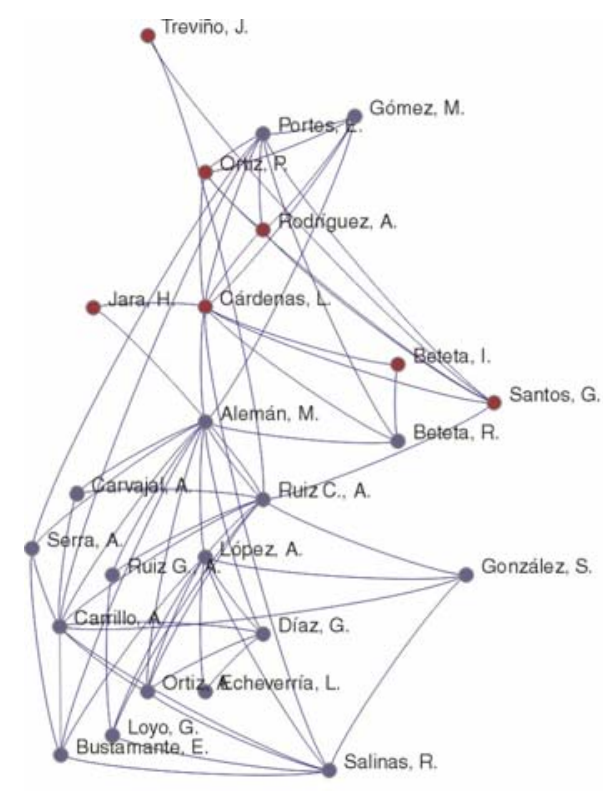

1960

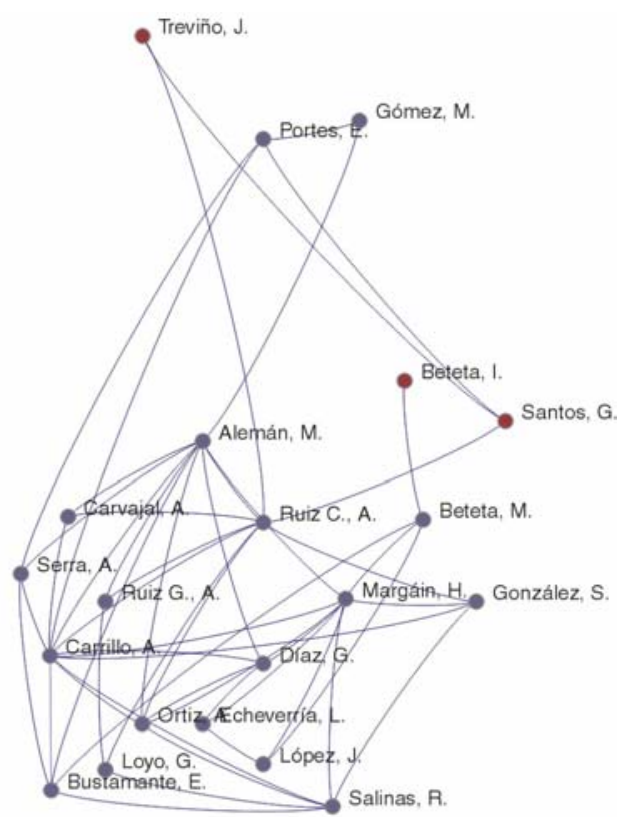

1970

Figura $6 \mathrm{~b}$. La red a través del tiempo (militares-nodos de color rojo)

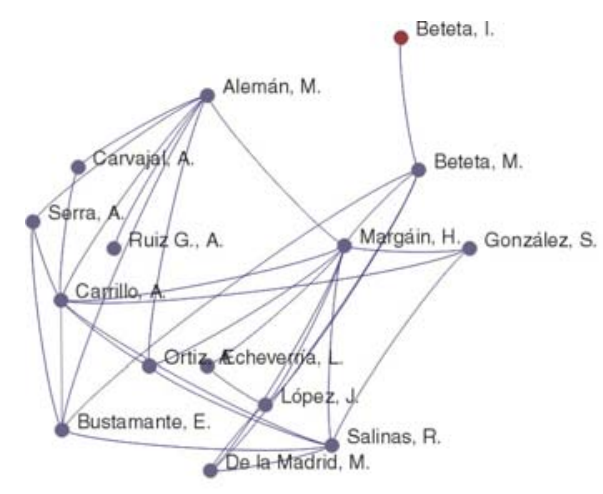

1980

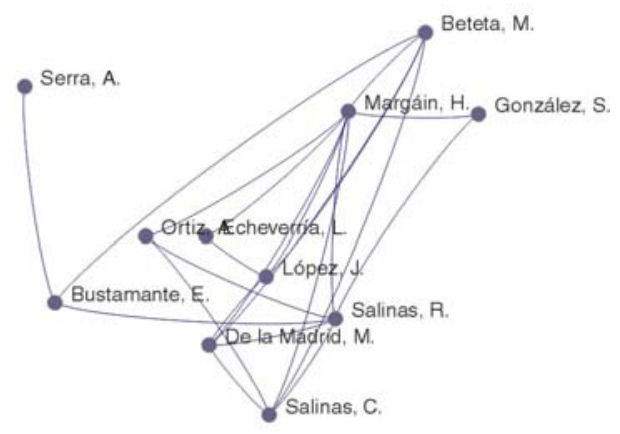

1990

Figura 6c. La red a través del tiempo (militares-nodos de color rojo)

En las Figuras 7a, 7b y 7c podemos observar el grado nodal para cada uno de los actores en cada corte, vemos que Miguel Alemán Valdés fue el único ex-presidente que tuvo un grado nodal mayor a los demás actores. Por su parte vemos que Hugo B. Margáin Gleason es el actor con mayor grado nodal en los dos últimos periodos. 


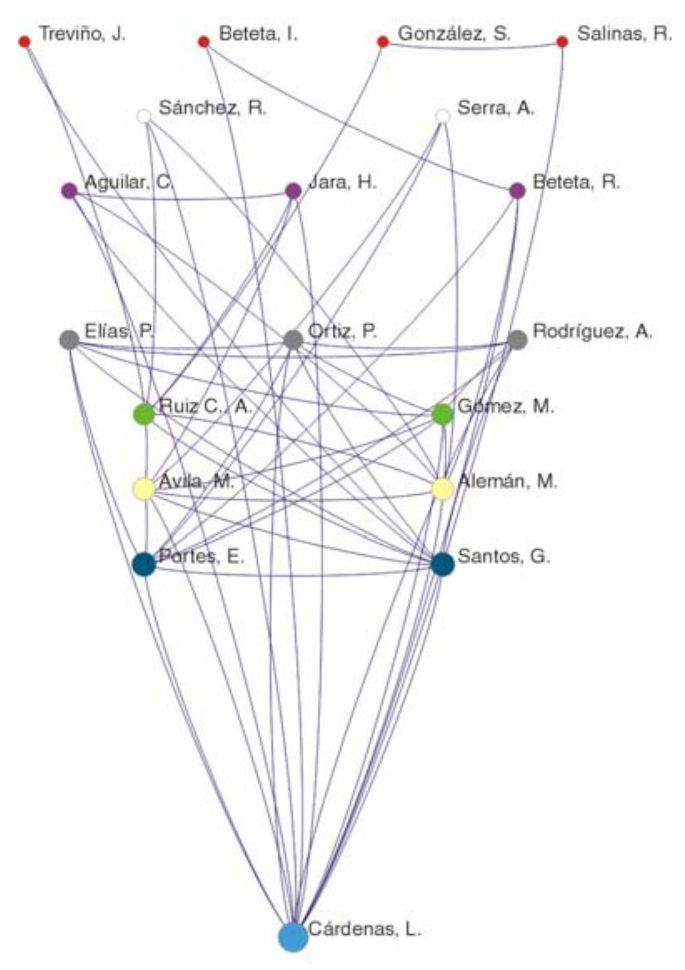

1940

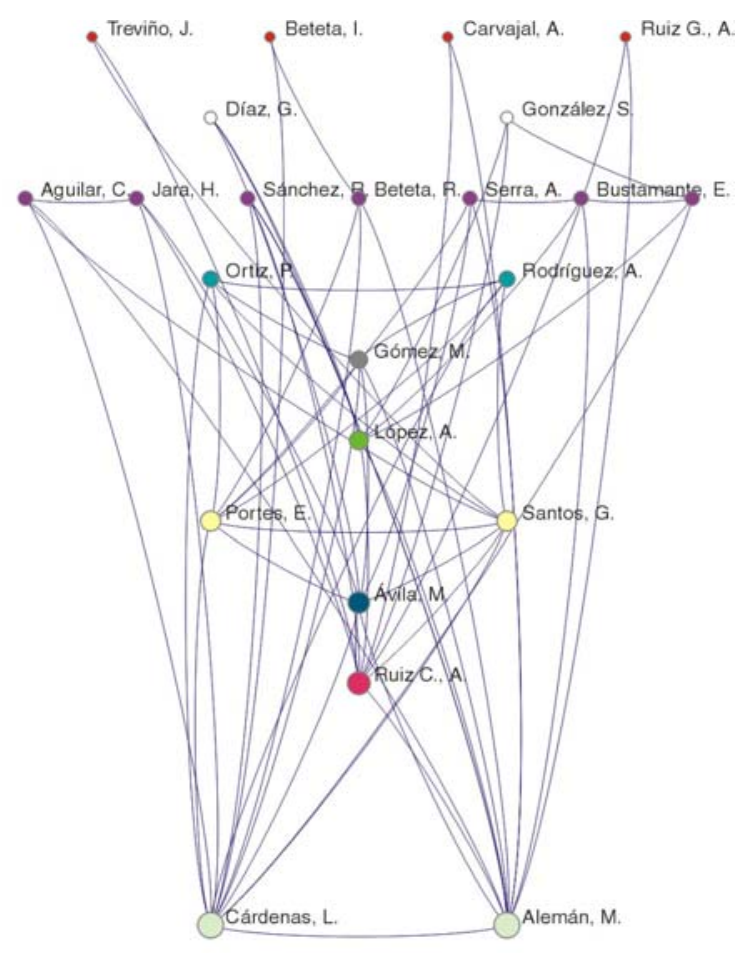

1950

Figura 7a. La red a través del tiempo (grado nodal)

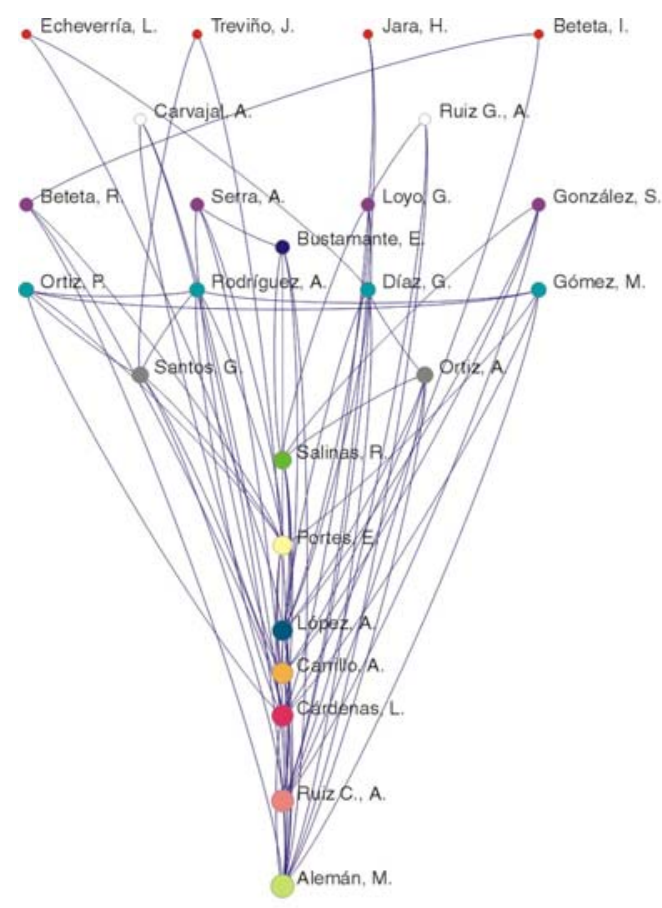

1960

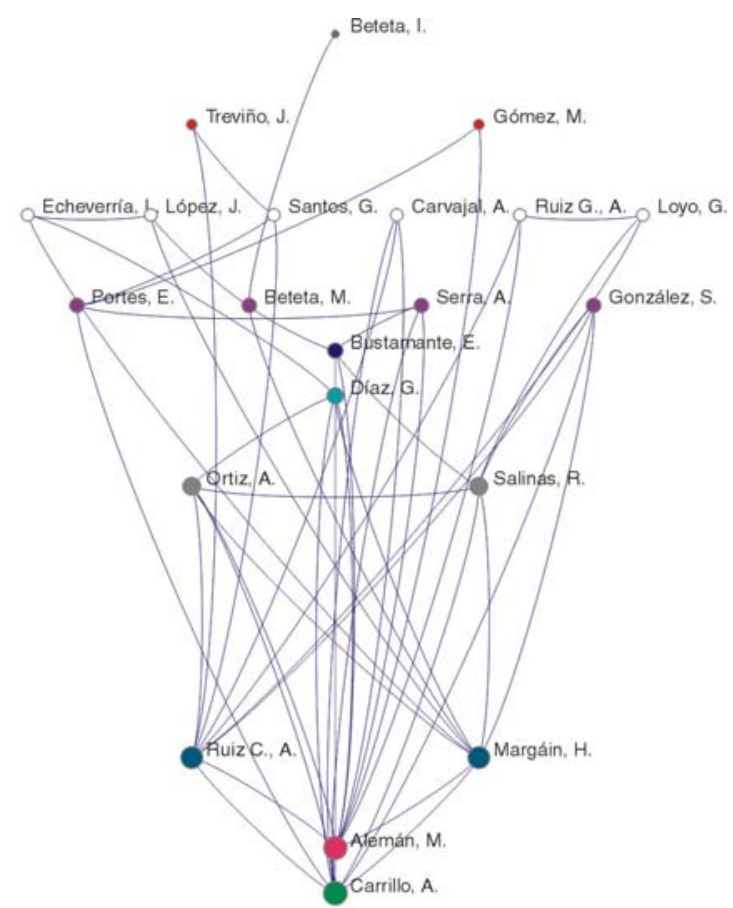

1970

Figura 7b. La red a través del tiempo (grado nodal) 


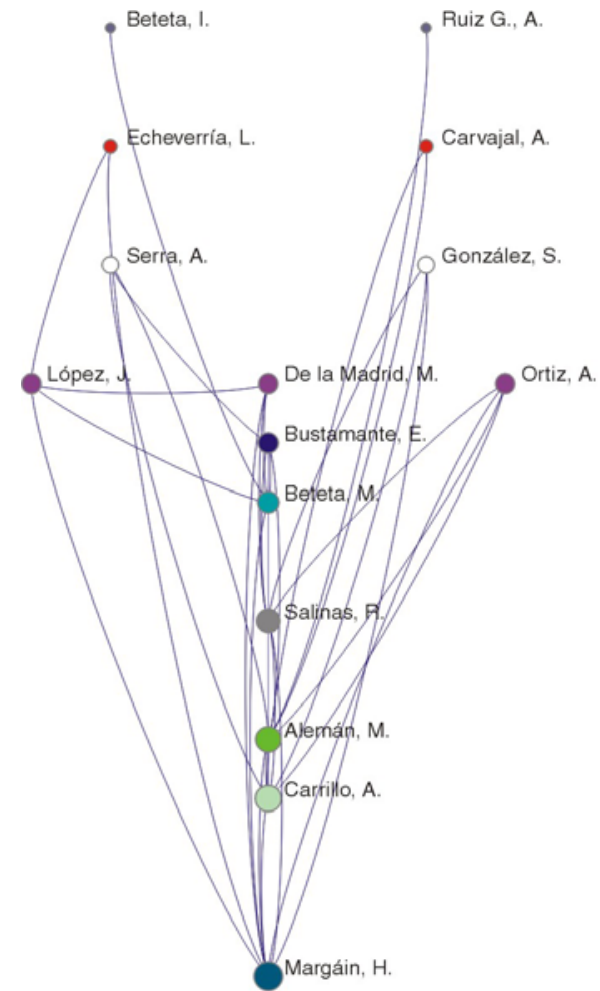

1980

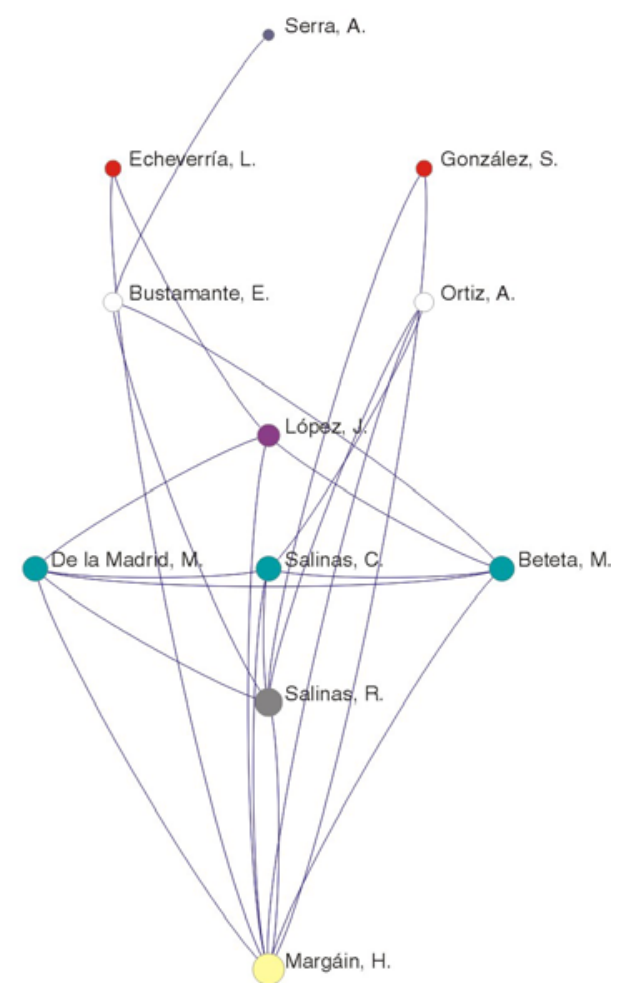

1990

Figura 7c. La red a través del tiempo (grado nodal)

\section{El grupo de los ex-presidentes}

Analizando las conexiones entre los ex-presidentes es claro que históricamente algunos no coincidieron ${ }^{3}$ (Figura 8), sin embargo al observar las redes por periodo es muy notorio el cambio que se da a través del tiempo. En la Figura 9 se muestra una disminución de relaciones entre los ex-presidentes de épocas recientes, al grado de la casi inexistente relación entre Carlos Salinas de Gortari y su predecesor Ernesto Zedillo Ponce de León. Esto puede servirnos como indicador del deterioro presidencial.

${ }^{3}$ Ibíd. p 106 


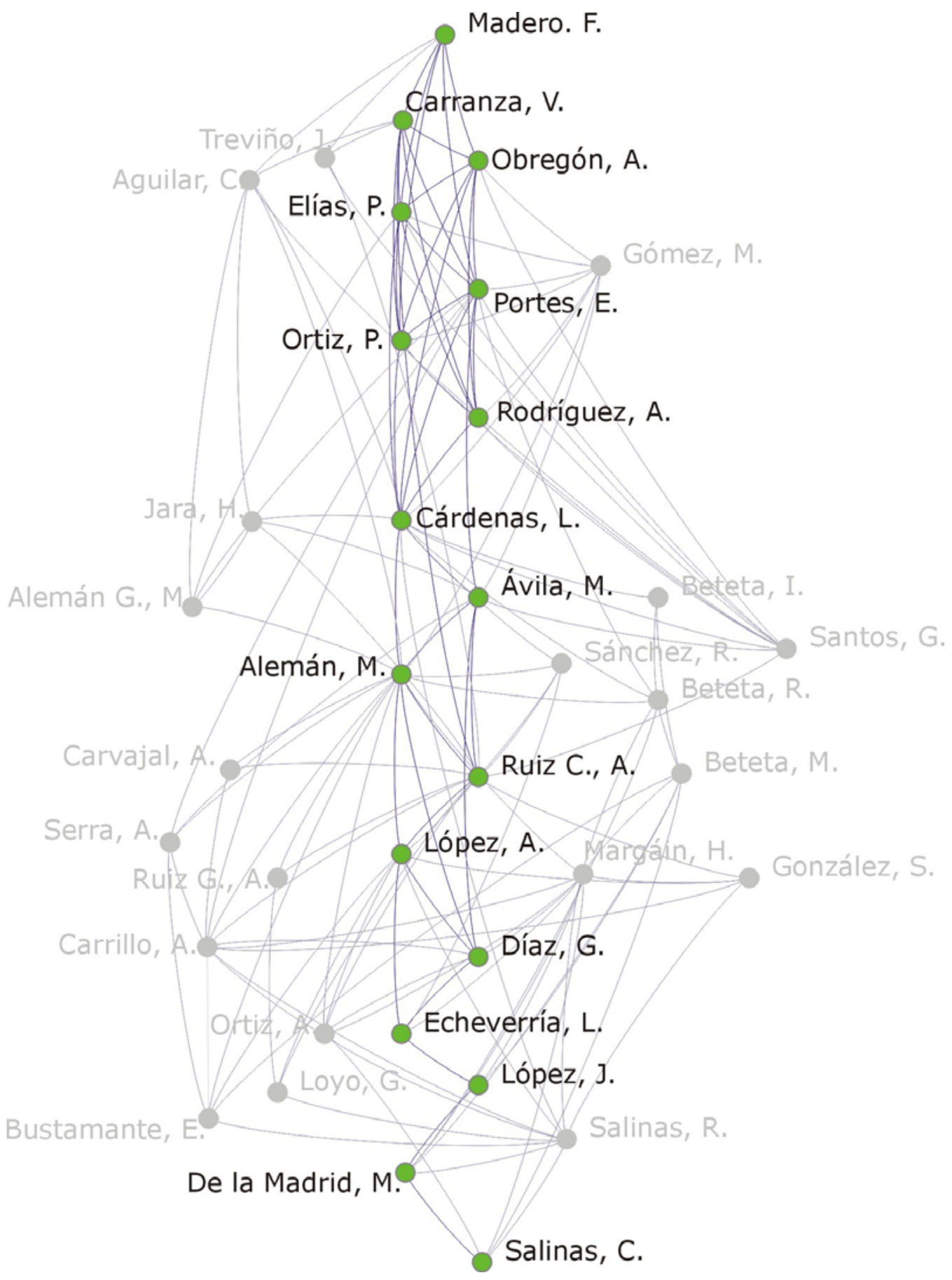

Figura 8. Ex-presidentes 


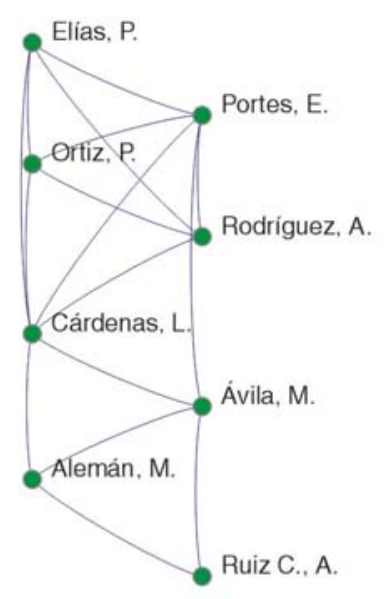

1940

- Portes, E.

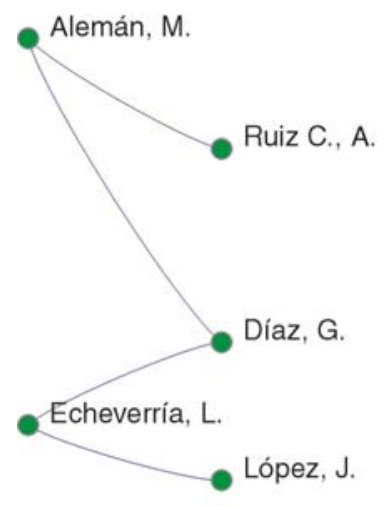

1970

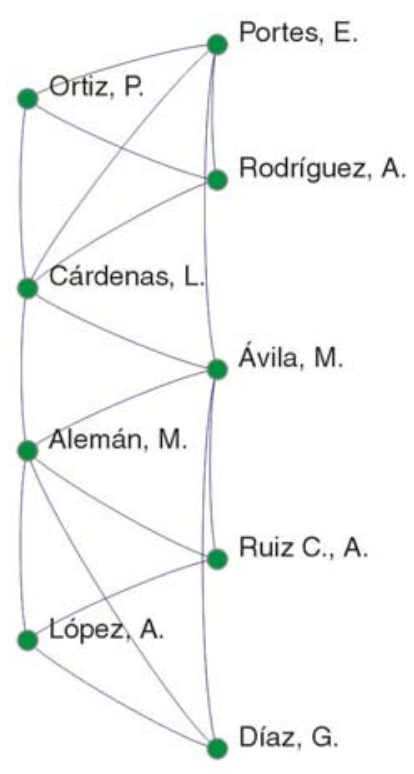

1950

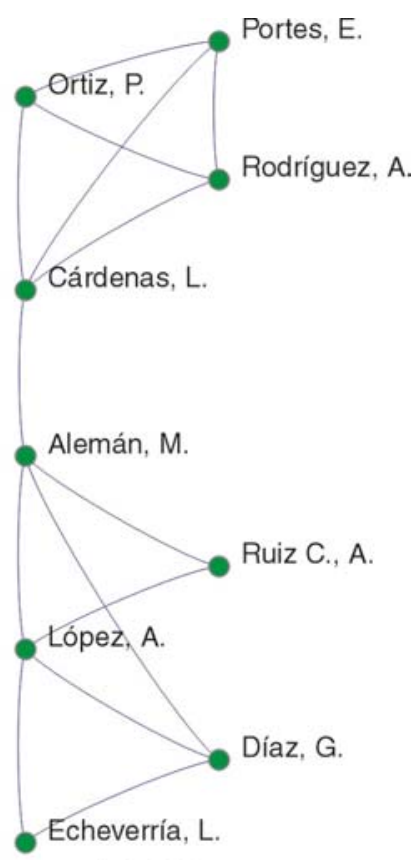

1960

- Alemán, M.

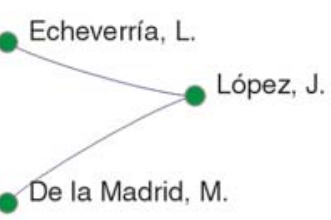

1980

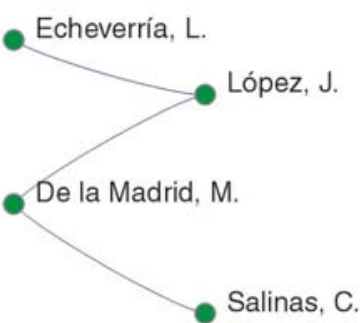

1990

Figura 9. Ex-presidentes

En su trabajo Gil-Mendieta y Schmidt establecen que el decrecimiento del índice de centralidad de los nodos resulta en menos integración y cohesión de la red, y en el caso de los ex-presidentes esto sugiere un debilitamiento en su rol. 
"La red de poder mexicana logró una gran cohesión que con el paso de los años se ha ido minando. Los actores políticos contemporáneos han perdido cohesión, lo cual se muestra con valores de centralidad más bajos de los alcanzados por los actores que iniciaron la red ... En México dos grupos de la red de poder han dominado al sistema político desde 1920, compitiendo entre ellos y definiendo sus trincheras alrededor de funciones sistémicas. Uno de los grupos está identificado con antecedentes militar-revolucionarios y posiblemente por eso con una agenda social muy clara, y el otro con el manejo de las finanzas, por lo cual seguramente ven la función gubernamental como una cuestión de eficiencia. ${ }^{\prime 4}$

En las figuras podemos observar a los actores con una gran permanencia dentro de la red, el cambio generacional que se da entre el año de 1970 y el año de 1980, cuales son los actores con mayor grado nodal en diferentes momentos y la relación entre el subgrupo de los ex-presidentes a través de tiempo. Cabe señalar que si bien las figuras pueden usarse como herramienta exploratoria, son la visualización de la composición y estructura de la red en un momento o periodo determinado.

\section{Referencias}

Wasserman, S. and K. Faust (1994). Social Network Analysis: methods and applications, Cambridge.

Everett, M. G. and S. P. Borgatti (1998). "Analyzing Clique Overlap". CONNECTIONS 21(1):49-61. 1998.

Schmidt, S. y Gil-Mendieta, J. (2002). "La red de poder mexicana" en Análisis de Redes: Aplicaciones en Ciencias Sociales, Jorge Gil Mendieta y Samuel Schmidt (eds). IIMAS. UNAM. pp 95-156.

Batagelj, V. and A. Mrvar. Pajek: Program for Analysis and Visualization of Large Networks, Reference Manual, V 1.0, 2004.

http://vlado.fmf.uni-lj.si/pub/networks/pajek/default.htm

\footnotetext{
${ }^{4}$ Ibíd. p 127
} 


\section{Anexo1. Los 37 actores}

\begin{tabular}{|c|c|c|c|c|}
\hline 1 & Madero. F. & Madero González, Francisco I. & ex presidente & civil \\
\hline 2 & Carranza, V. & Carranza Garza, Venustiano & ex presidente & militar \\
\hline 3 & Obregón, A. & Obregón Salido, Alvaro & ex presidente & militar \\
\hline 4 & Elías, $\mathrm{P}$. & Elías Calles, Plutarco & ex presidente & militar \\
\hline 5 & Portes, E. & Portes Gil, Emilio & ex presidente & civil \\
\hline 6 & Ortiz, P. & Ortiz Rubio, Pascual & ex presidente & militar \\
\hline 7 & Rodríguez, A. & Rodríguez Lujan, Abelardo L. & ex presidente & militar \\
\hline 8 & Cárdenas, L. & Cárdenas del Río, Lázaro & ex presidente & militar \\
\hline 9 & Ávila, M. & Ávila Camacho, Manuel & ex presidente & militar \\
\hline 10 & Alemán, M. & Alemán Valdés, Miguel & ex presidente & civil \\
\hline 11 & Ruiz C., A. & Ruiz Cortines, Adolfo & ex presidente & civil \\
\hline 12 & López, A. & López Mateos, Adolfo & ex presidente & civil \\
\hline 13 & Díaz, G. & Díaz Ordaz, Gustavo & ex presidente & civil \\
\hline 14 & Echeverría, L. & Echeverría Álvarez, Luis & ex presidente & civil \\
\hline 15 & López, J. & López Portillo, José & ex presidente & civil \\
\hline 16 & De la Madrid, $\mathrm{M}$. & De la Madrid Hurtado, Miguel & ex presidente & civil \\
\hline 17 & Salinas, C. & Salinas de Gortari, Carlos & ex presidente & civil \\
\hline 18 & Aguilar, C. & Aguilar Vargas, Cándido & & militar \\
\hline 19 & Treviño, J. & Treviño González, Jacinto B. & & militar \\
\hline 20 & Gómez, M. & Gómez Segura, Marte R. & & civil \\
\hline 21 & Santos, G. & Santos Rivera, Gonzalo N. & & militar \\
\hline 22 & Alemán G., M. & Alemán González, Miguel & & militar \\
\hline 23 & Jara, $\mathrm{H}$. & Jara Rodríguez, Heriberto & & militar \\
\hline 24 & Beteta, I. & Beteta Quintana, Ignacio & & militar \\
\hline 25 & Sánchez, R. & Sánchez Taboada, Rodolfo & & militar \\
\hline 26 & Beteta, R. & Beteta Quintana, Ramón & & civil \\
\hline 27 & Beteta, M. & Beteta Molsalve, Mario Ramón & & civil \\
\hline 28 & Carvajal, A. & Carvajal Bernal, Ángel & & civil \\
\hline 29 & Serra, A. & Serra Rojas, Andrés & & civil \\
\hline 30 & Ruiz G., A. & Ruiz Galindo, Antonio & & civil \\
\hline 31 & Carrillo, A. & Carrillo Flores, Antonio & & civil \\
\hline 32 & Bustamante, E. & Bustamante Vasconcelos, Eduardo & & civil \\
\hline 33 & Loyo, G. & Loyo González, Gilberto & & civil \\
\hline 34 & Ortiz, A. & Ortiz Mena, Antonio & & civil \\
\hline 35 & Margáin, $\mathrm{H}$. & Margáin Gleason, Hugo B. & & civil \\
\hline 36 & González, S. & González Blanco, Salomón & & civil \\
\hline 37 & Salinas, R. & Salinas Lozano, Raúl & & civil \\
\hline
\end{tabular}

